\title{
Insensibilidad congénita al dolor.
} Congenital insensitivity to pain.

Erika Palacios Rosas Ricardo Enrique Buendía Corona²

1 y 2 Médico general. Trabajador independiente, San José Costa Rica.

Contactos: erika.palacios@udlap.mx ricardo.buendia96@gmail.com

\section{RESUMEN}

El dolor representa una serie de reacciones en cadena resultado de un estímulo externo interno como una señal de alerta para el individuo que ha sufrido algún daño poniendo en riesgo su estabilidad física; sin embargo, existe una pequeña fracción de la población que ha nacido con alteraciones genéticas que les imposibilitan experimentar tal sensación, lo que repercute en el desarrollo pleno y la constante autolesión involuntaria. Este trabajo pretende dar un panorama conciso basado en evidencias sobre la etiología, epidemiología, signos y síntomas, el diagnóstico y los posibles tratamientos de la rara enfermedad denominada "Insensibilidad Congénita al Dolor" o ICD.

Palabras Clave: Insensibilidad congénita al dolor, atributos de enfermedad, enfermedades raras, enfermedades congénitas.

\section{ABSTRACT}

Pain represents a series of chain reactions from whether an extern or intern stimulus as an alert signal for the patient that has suffered damage putting his physical condition at risk. Nonetheless, there is a small fraction of the population that is

Cómo citar:

Palacios-Rosas, E., Buendía-Corona, R. E., \& Buendía-Corona, R. E. Insensibilidad congénita al dolor. Revista Ciencia Y Salud Integrando Conocimientos, 5(4). https://doi. org/10.34192/

cienciaysalud.v5i4.314 born with genetic alterations that make it impossible for them to experience such sensation, which affects full development and constant involuntary self-harm. The intend of this review is to give a concise based on evidence panorama about the etiology, epidemiology, signs and symptoms, diagnosis and possible treatments for the rare disease named "Congenital Insensitivity to Pain" or ICD..

Keywords: Congenital pain insensitivity, disease attributes, rare disease, congenital.

Recibido: 11/may/2021

Aceptado: 28/ago/2021

Publicado: 04/sep/2021

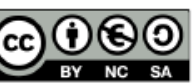




\section{INTRODUCCIÓN}

La Insensibilidad Congénita al Dolor (ICD) es considerada una enfermedad rara descrita por primera vez por Van Ness Dearborn en 19321 como "analgesia pura congénita"; a pesar de ser una enfermedad conocida desde hace varios años, durante el 2020 solo se han reportado 20 casos en la literatura científica2. Se trata de un padecimiento en el cual el paciente es incapaz de percibir dolor a partir de estímulos, incluida la inflamación y el calor; este tipo de alteración obtenida desde el nacimiento puede conllevar a la continua aparición de lesiones y/o la nula recuperación de las mismas3. Está catalogada como una neuropatía autosomal recesiva4; es decir, deben existir afectaciones en ambos alelos para presentar estos fenotipos, dichas alteraciones son ocasionadas en múltiples genes relacionados al dolor como canales iónicos, mielinización, desarrollo neuronal, entre otros5,6.

\section{MATERIALES Y MÉTODOS}

Se llevó a cabo un estudio de revisión bibliográfica, utilizando el motor de búsqueda PubMed, OMIM, y Uniprot. Para la evaluación de estudios consultados no se estableció un filtro de año de publicación dado que el objetivo de la revisión fue establecer un panorama histórico. Los criterios de inclusión fueron el análisis de artículos originales y revisiones sistemáticas publicados en las bases de datos mencionados con los siguientes términos de selección: Congenital AND Insensivity to pain.

\section{ETIOLOGÍA}

Recientes estudios han catalogado la presencia de siete genes implicados en el desarrollo de ICD7-12; sin embargo, esta sección del artículo se enfocará en describir dos de ellos: Receptor de alta afinidad del factor de crecimiento nervioso (NTRK1, Figura 1A) y la proteína subunidad alfa tipo 9 del canal de sodio (SCN9A, Figura 1B), los cuales son catalogados como los genes cuyas alteraciones son más comunes en los casos de ICD3.

A)

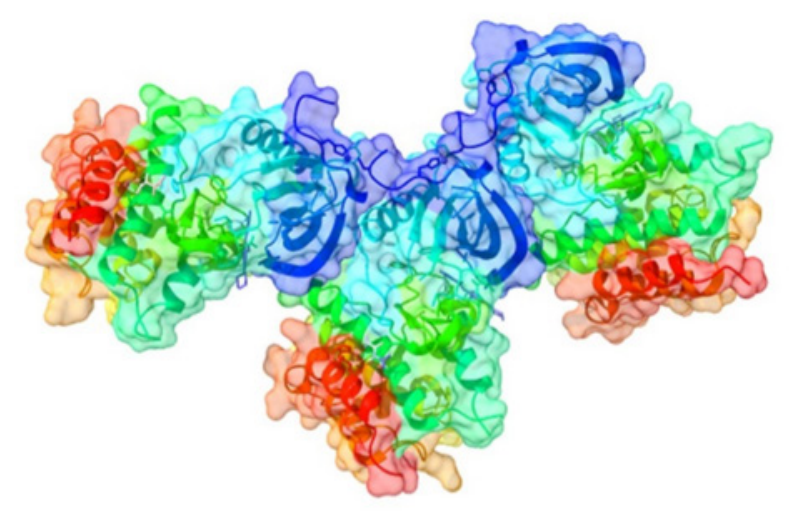

B)

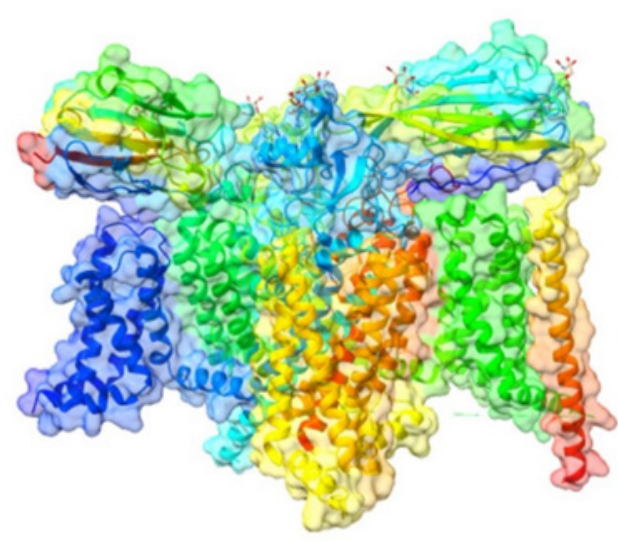

Figura 1. Representación tridimensional de los genes asociados a la ICD. A) NTRK1 (PDB ID: 6PME) y B) SCN9A (PDB ID: 6J8J). Imágenes generadas en el software Chimera X13.

NTRK1: participa en el desarrollo y maduración de los sistemas nerviosos central y periférico mediante la regulación de la proliferación, diferenciación y supervivencia de las neuronas simpáticas y nerviosas mediante la fosforilación del factor de crecimiento nervioso (NGF) encargado del crecimiento neuronal en etapa fetal14. Mutaciones en este gen desembocan en la pérdida de comunicación entre las neuronas y el órgano en que desembocan15 y al menos 30 variaciones (sustituciones/deleciones) en la secuencia nativa han sido descritas y correlacionadas a ICD en la base de datos UniProt (ID: P04629). 
SCN9A: Media la permeabilidad de iones de sodio dependiente del voltaje de las membranas excitables, asumiendo distintas conformaciones en respuesta al voltaje por el cual los iones pasan de acuerdo con el gradiente electroquímico, lo que lo envuelve en el rol del desarrollo del dolor16-17. Variaciones en las posiciones 907 y 1381 - 1385 han sido descritas y correlacionadas a ICD en la base de datos UniProt (ID: Q15858).

\section{EPIDEMIOLOGÍA}

Esta enfermedad es categorizada como rara debido a la poca prevalencia que muestra, en 2007 se tenía un estimado de 1 persona por cada 2500018; sin embargo, el estimado cambia para el año 2020 con un total de 1 en 1000000 de personas3. La enfermedad ha sido más conocida por casos aislados reportados en canales de televisión o redes sociales19,20.

\section{SIGNOS Y SÍNTOMAS}

El síntoma recurrente en todos los casos es la ausencia de sensación de dolor, no importa el estímulo, su gravedad o su duración, el paciente es incapaz de percatarse de lo ocurrido hasta tener visualización directa de la lesión11. Aunado a esto, cada paciente registrado en la literatura científica ha presentado un cuadro clínico variado dependiendo de la historia y experiencias que lo preceden como se puede visualizar en la tabla 1.

Tabla 1. Signos más comunes asociados a ICD.

\begin{tabular}{|c|c|}
\hline Signos & Referencia \\
\hline $\begin{array}{c}\text { Automutilación en los dedos, lengua y pérdida de labios } \\
\text { inferiores }\end{array}$ & 3 \\
\hline $\begin{array}{l}\text { Otitis recurrente por inmunidad reducida a } \\
\text { Staphylococcus aureus }\end{array}$ & 21 \\
\hline $\begin{array}{c}\text { Quemaduras debido a alteración en la sensación de } \\
\text { temperaturas }\end{array}$ & 16 \\
\hline Fracturas y deformidades progresivas & 22 \\
\hline $\begin{array}{l}\text { Anhidrosis (falta de sudor) lo cual desemboca en fiebres } \\
\text { inexplicables }\end{array}$ & 23 \\
\hline $\begin{array}{cc}\text { Hiperhidrosis } \begin{array}{c}\text { (exceso de sudor) en aquellos con } \\
\text { patogenia heterocigota }\end{array} & \end{array}$ & 24 \\
\hline Inteligencia & 3 \\
\hline $\begin{array}{c}\text { Aquellos con alteración en NTRK1 desarrollan cierta } \\
\text { discapacidad intelectual. } \\
\text { Aquellos con alteración en SCN9A típicamente tienen } \\
\text { inteligencia normal. }\end{array}$ & \\
\hline En algunos casos es posible detectar anemia crónica & 21 \\
\hline
\end{tabular}

\section{DIAGNÓSTICO}

Existe mucha controversia entre los distintos métodos de diagnóstico utilizados ya que se pone a prueba la sensación del dolor mediante la estimulación de este; a pesar de que el análisis genético de las variantes génicas NTRK1 y SCN9A, entre otros genes, son las pruebas estándar de oro, su accesibilidad aún se ve limitada por recursos económicos y/o capacitación técnica de laboratorio, debido a esto, se enlista en la tabla 2 una serie de procedimientos de apoyo para el diagnóstico oportuno, cabe destacar que un paciente con ICD es negativo a las pruebas descritas. 


\section{CIENCIA\&SALUD \\ Ucim:o}

Tabla 2. Pruebas de diagnóstico para ICD.

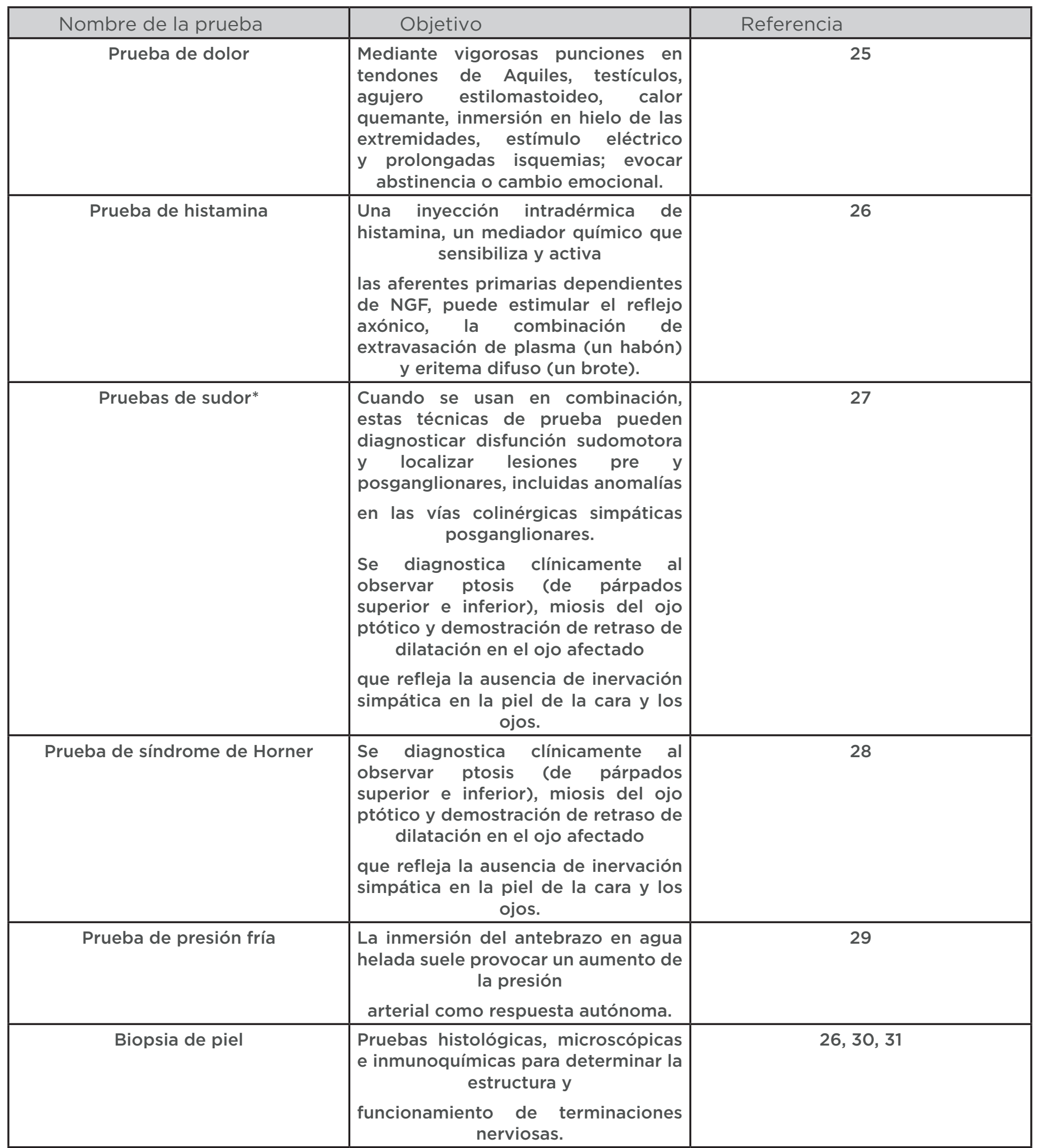

*Se incluyen: prueba termoregulatoria, prueba cuantitativa del reflejo axónico sudomotor, respuesta cutánea 


\section{CIENCIA\&SALUD}

simpática, prueba de acetilcolina en las manchas de sudor y prueba cuantitativa de reflejo axónico directo e indirecto.

\section{TRATAMIENTO}

Se ha propuesto el uso de nalaxona; un derivado de la tebaína, que revierte de forma antagonista los efectos de los analgésicos opioides al unirse a sus receptores en el sistema nervioso central32; esto quiere decir que como medicamento alivia la insensibilidad de los pacientes (provoca/revela dolor); sin embargo, este solo puede usarse para localizar la región ya dañada o enfermedad desarrollada e incluso podría revelar múltiple dolor debido a lesiones acumuladas33.

La ICD solía ser catalogada como una enfermedad de niños, ya que, tras desatender las lesiones, tardío diagnóstico, lenta recuperación, la expectativa de vida era de 25 años de edad como máximo34; no obstante, el paciente puede lograr llegar a la adultez sin representar mayor problema bajo un cuidado médico apropiado, un seguimiento y evaluación constante con la intervención de especialistas que cubran las áreas más comunes y afectadas; dentistas, dermatólogos, oftalmólogos, endocrinólogos, ortopedistas, recurrentes análisis químicos, hormonales, control de la temperatura e incluso cuidado de la integridad mental35.

Como se describe en la etiología de la enfermedad, existen al menos siete genes implicados en el desarrollo de la enfermedad, lo cual dificulta incluso la posibilidad de contar con un mecanismo de terapia génica; la poca prevalencia de la enfermedad dificulta el desarrollo de un tamizaje neonatal genético estándar.

\section{CONCLUSIONES}

La ICD representa un problema de salud menor debido a la poca incidencia que representa mundialmente; conocer acerca de los factores genéticos que provocan el desarrollo de la enfermedad ha ayudado a entender cómo funciona el dolor tanto en personas sanas como en afectadas por la insensibilidad al mismo. Si bien no es posible revertir todas y cada una de las mutaciones que provocan el padecimiento, es posible desarrollar fármacos que revelen por un tiempo la ubicación de zonas dañadas para mejorar los tratamientos que permiten sobrellevar la enfermedad; además, el conocimiento sobre las proteínas implicadas en la ICD puede funcionar como una oportunidad para desarrollar medicamentos que sean más capaces de controlar el dolor crónico y agudo, ofreciendo una oportunidad de entender al dolor, cuándo, cómo y por qué inhibirlo.

\section{REFERENCIAS BIBLIOGRÁFICAS}

1. Van Ness Dearborn G. A case of congenital general pure analgesia. J Nerv Ment Dis. 1932;75(6):612-5. DOI: 10.1097/00005053-193206000-00002.

2. MedlinePlus. Congenital insensitivity to pain [Internet]. Genetic Conditions. 2020 [cited 2021 Feb20]. Available from: https://medlineplus.gov/genetics/condition/congenitalinsensitivity-to- pain/\#frequency

3. Adam M, Ardinger H, Pagon R. Congenital Insensitivity to Pain Overview. In: GeneReviews [Internet]. Seattle; 2021 [cited 2021 Feb 20]. Available from: https://www.ncbi.nlm.nih.gov/books/ NBK481553/

4. Scott BK, Baranov D. Neurologic Diseases. In: Anesthesia and Uncommon Diseases: Sixth Edition. 6th ed. Elsevier Inc.; 2012. p. 251-95. DOI: 10.1016/B978-1-4377-2787-6.00008-5.

5. Landrieu P, Baets J. Early onset (childhood) monogenic neuropathies. In: Handbook of Clinical Neurology. Elsevier B.V.; 2013. p. 863-91. DOI: 10.1016/B978-0-444-52902-2.00049-7. 
6. Klein CJ, Dyck PJ. HSANs: Clinical Features, Pathologic Classification, and Molecular Genetics. In: Peripheral Neuropathy. Elsevier Inc.; 2005. p. 1809-44. DOI: 10.1016/B978-0-7216-9491-7.50081-8.

7. Shaikh SS, Nahorski MS, Woods CG. A third HSAN5 mutation disrupts the nerve growth factor furin cleavage site. Mol Pain [Internet]. 2018 Oct 1 [cited 2021 Feb 2O];14. DOI: 10.1177/1744806918809223.

8. Indo Y, Tsuruta M, Hayashida Y, Karim MA, Ohta K, Kawano T, et al. Mutations in the TRKA/NGF receptor gene in patients with congenital insensitivity to pain with anhidrosis. Nat Genet [Internet]. 1996 [cited 2021 Feb 20];13(4):485-8. DOI: 10.1038/ng0896-485.

9. Zhang S, Sharif SM, Chen YC, Valente EM, Ahmed M, Sheridan E, et al. Clinical features for diagnosis and management of patients with PRDM12 congenital insensitivity to pain. J Med Genet [Internet]. 2016 Aug 1 [cited 2021 Feb 20];53(8):533-5. DOI: 10.1136/jmedgenet-2015-103646.

10. Cox JJ, Sheynin J, Shorer Z, Reimann F, Nicholas AK, Zubovic L, et al. Congenital insensitivity to pain: Novel SCN9A missense and in-frame deletion mutations. Hum Mutat [Internet]. 2010 Sep [cited 2021 Feb 20];31(9):1670. DOI: 10.1002/humu.21325.

11. Phatarakijnirund V, Mumm S, McAlister WH, Novack D V., Wenkert D, Clements KL, et al. Congenital insensitivity to pain: Fracturing without apparent skeletal pathobiology caused by an autosomal dominant, second mutation in SCN11A encoding voltage-gated sodium channel 1.9. Bone [Internet]. 2016 Mar 1 [cited 2021 Feb 20];84:289-98. DOI: 10.1016/j.bone.2015.11.022.

12. Habib AM, Matsuyama A, Okorokov AL, Santana-Varela S, Bras JT, Aloisi AM, et al. A novel human pain insensitivity disorder caused by a point mutation in ZFHX2. Brain [Internet]. 2018 Feb 1 [cited 2021 Feb 20];141(2):365-76. DOI: 10.1093/brain/awx326.

13. Pettersen EF, Goddard TD, Huang CC, Meng EC, Couch GS, Croll TI, et al. UCSF ChimeraX: Structure visualization for researchers, educators, and developers. Protein Sci [Internet]. 2021 Jan 1 [cited 2021 Feb 20];30(1):70-82. DOI: 10.1002/pro.3943.

14. Jing S, Tapley P, Barbacid M. Nerve growth factor mediates signal transduction through trk homodimer receptors. Neuron. 1992;9(6):1067-79. DOI: 10.1016/0896-6273(92)90066-M.

15. Nam TS, Li W, Yoon S, Eom GH, Kim MK, Jung ST, et al. Novel NTRK1 mutations associated with congenital insensitivity to pain with anhidrosis verified by functional studies. J Peripher Nerv Syst [Internet]. 2017 Jun 1 [cited 2021 Feb 20];22(2):92-9. DOI: 10.1111/jns.12205.

16. Cox JJ, Reimann F, Nicholas AK, Thornton G, Roberts E, Springell K, et al. An SCN9A channelopathy causes congenital inability to experience pain. Nature. 2006 Dec 14;444(7121):894-8. DOI: 10.1038/nature05413.

17. Alles SRA, Nascimento F, Luján R, Luiz AP, Millet Q, Ali Bangash M, et al. Sensory neuronderived NaV1.7 contributes to dorsal horn neuron excitability. Sci Adv [Internet]. 2020 [cited 2021 Feb 20];6(8):4568. DOI: 10.1126/sciadv.aax456.

18. Sasnur AH, Sasnur PA, Ghaus-UI RSM. Congenital insensitivity to pain and anhidrosis. Indian J Orthop [Internet]. 2011 May [cited 2021 Feb 20];45(3):269-71. DOI: 10.4103/0019-5413.80047.

19. 60 Minutes Australia. Liz Hayes talking about children with congenital insensitivity to pain. [Internet]. EXTRA MINUTES. 2013 [cited 2021 Feb 20]. Available from: https://www.youtube.com/ 


\section{CIENCIA\&SALUD}

watch?v=03U-fGj17go

20. ABC News. Young Girl Cannot Feel Pain, Battles Rare Medical Condition CIPA [Internet]. Good Morning America. 2012 [cited 2021 Feb 20]. Available from: https://www.youtube.com/ watch?v=n6iOUW523BE

21. Shatzky S, Moses S, Levy J, Pinsk V, Hershkovitz E, Herzog L, et al. Congenital insensitivity to pain with anhidrosis (CIPA) in Israeli- Bedouins: Genetic heterogeneity, novel mutations in the TRKA/NGF receptor gene, clinical findings, and results of nerve conduction studies. Am J Med Genet [Internet]. 2000 Jun 19 [cited 2021 Feb 20];92(5):353-60. DOI: 10.1002/1096-8628(20000619)92:5<353::AID-AJMG12>3.0.CO;2-C.

22. Staudt MD, Bailey CS, Siddiqi F. Charcot spinal arthropathy in patients with congenital insensitivity to pain: a report of two cases and review of the literature [Internet]. Vol. 41, Neurosurgical Review. Springer Verlag; 2018 [cited 2021 Feb 20]. p. 899-908. DOI: 10.1007/s10143-017-0814-3.

23. Indo Y, Mardy S, Miura Y, Moosa A, Ismail EAR, Toscano E, et al. Congenital insensitivity to pain with anhidrosis (CIPA): Novel mutations of the TRKA (NTRK1) gene, a putative uniparental disomy, and a linkage of the mutant TRKA and PKLR genes in a family with CIPA and pyruvate kinase deficiency. Hum Mutat. 2001;18(4):308-18. DOI: 10.1002/humu.1192.

24. Woods CG, Babiker MOE, Horrocks I, Tolmie J, Kurth I. The phenotype of congenital insensitivity to pain due to the Na v 1.9 variant p.L811P [Internet]. Vol. 23, European Journal of Human Genetics. Nature Publishing Group; 2015 [cited 2021 Feb 20]. p. 561-3. DOI: 10.1038/ejhg.2014.166.

25. Swanson AG. Congenital Insensitivity to Pain with Anhydrosis: A Unique Syndrome in Two Male Siblings. Arch Neurol [Internet]. 1963 [cited 2021 Mar 8];8(3):299-306. DOI: 10.1001/ archneur.1963.00460030083008.

26. Indo Y. Genetics of congenital insensitivity to pain with anhidrosis (CIPA) or hereditary sensory and autonomic neuropathy type IV: Clinical, biological and molecular aspects of mutations in TRKA(NTRK1) gene encoding the receptor tyrosine kinase for nerve growth factor. Clin Auton Res [Internet]. 2002 [cited 2021 Mar 8];12(1 SUPPL.). DOI: 10.1007/s102860200016.

27. Illigens BMW, Gibbons $\mathrm{CH}$. Sweat testing to evaluate autonomic function [Internet]. Vol. 19, Clinical Autonomic Research. NIH Public Access; 2009 [cited 2021 Mar 8].p. 79-87. DOI: 10.1007/ s10286-008-0506-8.

28. Brown JW, Podosin R. A Syndrome of the Neural Crest. Arch Neurol [Internet]. 1966 [cited 2021 Mar 8];15(3):294-301. DOI: 10.1001/archneur.1966.00470150072012.

29. Pinsky L, DiGeorge AM. Congenital familial sensory neuropathy with anhidrosis. J Pediatr. 1966 Jan 1;68(1):1-13. DOI: 10.1016/SOO22-3476(66)80417-1.

30. Nolano M, Crisci C, Santoro L, Barbieri F, Casale R, Kennedy WR, et al. Absent innervation of skin and sweat glands in congenital insensitivity to pain with anhidrosis. Clin Neurophysiol [Internet]. 2000 Sep 1 [cited 2021 Mar 8];111(9):1596-601. DOI: 10.1016/S1388-2457(00)00351-5.

31. Ismail EAR, Al-Shammari N, Anim JT, Moosa A. Congenital insensitivity to pain with anhidrosis: Lack of eccrine sweat gland innervation confirmed. J Child Neurol [Internet]. 1998 [cited 2021 Mar 


\section{CIENCIA\&SALUD}

8];13(5):243-6. DOI: 10.1177/088307389801300511.

32. National Center for Biotechnology Information. PubChem Compound Summary for CID 5284596, Naloxone. [Internet]. PubChem. 2021 [cited 2021 Mar 8]. Available from: https://pubchem. ncbi.nlm.nih.gov/compound/Naloxone

33. Minett MS, Pereira V, Sikandar S, Matsuyama A, Lolignier S, Kanellopoulos AH, et al. Endogenous opioids contribute to insensitivity to pain in humans and mice lacking sodium channel Nav1.7. Nat Commun [Internet]. 2015 Dec 4 [cited 2021 Feb 20];6. DOI: 10.1038/ncomms9967.

34. Daneshjou K, Jafarieh H, Raaeskarami SR. Congenital insensitivity to pain and anhydrosis (CIPA) syndrome; A report of 4 cases. Iran J Pediatr [Internet]. 2012 [cited 2021 Feb 20];22(3):412-6. Available from: /pmc/articles/PMC3564101/

35. NIH. Congenital insensitivity to pain with anhidrosis [Internet]. Genetic and Rare Diseases Information Center. 2020 [cited 2021 Feb 20]. Available from: https:// rarediseases.info.nih.gov/diseases/3006/congenital-insensitivity-to-pain- with-anhidrosis\#diseaseP rognosisSection 